\title{
Analysis of Serum Immune Markers in Seropositive and Seronegative Rheumatoid Arthritis Among Sudanese Patients and the Relation Between the Serotype and Joint Involvement: A Cohort Study
}

\author{
Ahmed Seri ${ }^{1,2}$ \\ Hala Kamal Ali Mohamed ${ }^{3-5}$ \\ Mohammed Elmujtba Adam \\ Essa (iD) ${ }^{6}$ \\ Elnour Mohammed Elagib ${ }^{7,8}$ \\ Noha Ibrahim Ahmed Eltahirm ${ }^{7,8}$ \\ Salma Mohammed Alfatih \\ Mansour ${ }^{9}$ \\ Abdelkareem A Ahmed $^{6}$ \\ 'Department of Clinical Immunology, \\ Royal Care International Hospital, \\ Khartoum, Sudan; ${ }^{2}$ Department of \\ Immunology, Institute of Endemic \\ Disease, University of Khartoum, \\ Khartoum, Sudan; ${ }^{3}$ Department of \\ Microbiology, Faculty of Medicine, African \\ International University (AIU), \\ Khartoum, Sudan; ${ }^{4}$ Department of \\ Clinical Immunology, Sudan Medical \\ Specialization Board (SMSB), Khartoum, \\ Sudan; ${ }^{5}$ Faculty of Medicine, Sudan \\ University of Science and Technology, \\ Khartoum, Sudan; ${ }^{6}$ Department of \\ Clinical Medicine, Medical and Cancer \\ Research Institute, Nyala, Sudan; \\ ${ }^{7}$ Department of Rheumatology, \\ Omdurman Military Hospital, Khartoum, \\ Sudan; ${ }^{8}$ Department of Medicine, Faculty \\ of Medicine, Karary University, \\ Khartoum, Sudan; ${ }^{9}$ Department of \\ Medicine, Faculty of Medicine, University \\ of Khartoum, Khartoum, Sudan
}

Correspondence: Mohammed Elmujtba Adam Essa

Department of Clinical Medicine, Medical and Cancer Research Institute, Nyala, Sudan

Tel +249 90700938

Email Awadali818@yahoo.com
Introduction: Rheumatoid arthritis (RA) is a chronic autoimmune disease characterized by inflammation of the synovial membrane. RA is classified as seropositive or seronegative, according to the absence or presence of primarily IgM RF, RF, and/or ACPA. The aim of this study is to identify the relationship between the serotype of rheumatoid arthritis and the level of ESR.

Methods and Materials: This is a descriptive, cross-sectional study done in Omdurman military hospital, Khartoum, Sudan. Conducted with 60 patients with RA, data were collected through a designated questionnaire which included demographic, age, gender, duration of the disease, laboratory finding. All the patients in the study were treated with conventional DMARDs and diagnosed according to the 2010 ACR/EULAR criteria; their disease activity status was assessed by DAS28/ESR. Data were analyzed using SPSS version 23.

Results: The study found that $91.7 \%$ of the patients were females, patients of age group between 36 and 50 years had the highest percentage at $38.3 \%$ followed by those between 51 and 70 years and the least age group between 20 and 35 years, $36.7 \%$ and $15 \%$, respectively. Of all the patients $61.7 \%$ were found to be SPRA, while the remaining $38.3 \%$ were seronegative (SNRA). Altogether $55 \%$ of the patients had moderate disease activity, followed by $16.7 \%$ who had a remission, $15 \%$ had high disease activity and the remaining $13.3 \%$ had low disease activity. The metacarpophalangeal (MCP) joint was found to be the only joint that was significantly associated with DAS28 and its involvement was greater among seropositive patients. The most affected joints were found to be shoulders, knees, wrist, MCP, PIP and elbow, in that order.

Conclusion: Females, middle-age group and shoulder joint were the most affected. Most RA was found to be SPRA, and the seropositive group was found to be more associated with high disease activity, while the seronegative group was associated with remission and low disease activity.

Keywords: seropositive, seronegative, RA, female, high disease activity

\section{Introduction}

Rheumatoid arthritis (RA) is a chronic, systemic inflammatory autoimmune disorder, in which the body's immune system mistakenly attacks the synovial joints, leading to joint inflammation which is a hallmark feature of this disease. ${ }^{1}$ RA is ranked as the 42nd highest contributor to global disability and its clinical features vary from small joint pain, swelling in a symmetrical manner, morning stiffness to 
extra-articular involvement such as the kidney, lungs, eyes and heart. ${ }^{2}$ The presence of autoantibodies (RF and ACPA and others) play an important role in immunopathogenesis of the condition through the formation of immune complexes on the synovial tissues, although, a recent report revealed the role of the aberrant cellular immune response as well, including Th1, Th2 and Th17 immune responses. $^{3,4}$

RA is now classified as seronegative or seropositive according to the absence or presence of anticitrullinated protein antibodies (ACPA) and rheumatoid factor (RF), and serological status has become significant in diagnosis, treatment, and prognosis decisions. ${ }^{5,6}$ Seronegative RA has been considered to represent a minimal severity when compared with seropositive RA, with less radiographic damage. 6,7 The presence of autoantibodies in early-stage RA has been associated with the development of comorbidities, such as pulmonary diseases and vasculitis. It has been reported that seropositive RA patients should be treated more aggressively based on the EULAR recommendations. ${ }^{8}$ Recent studies indicated that seronegative patients had significantly higher inflammatory activity at the time of diagnosis than seropositive patients. ${ }^{9}$ This study aims to compare Sudanese patients with seropositive and seronegative rheumatoid arthritis as well as to define the relationship between the serotype and particular joint involvement.

\section{Materials and Methods}

\section{Study Design}

A descriptive, non-interventional, analytical, cross-sectional, hospital-based study was conducted between October 2020 to December 2020.

\section{Study Area}

This study was conducted in the rheumatology clinic at Omdurman Military Hospital- Khartoum.

\section{Study Population}

Patients with rheumatoid arthritis presented to the rheumatology clinic at Omdurman Military Hospital during the time of the study.

\section{Inclusion Criteria}

- All patients diagnosed by rheumatologists with RA were included. A clinical "diagnosis" has to be established by the physician (rheumatologist) according to the 2010
ACR/European League Against Rheumatism (EULAR) criteria.

- Patients on conventional DMARDs were included.

\section{Exclusion Criteria}

- Patients who are not compliant to medications or off treatment (during the last 3 months).

- Patients who refused to participate in the study.

\section{Sampling}

Sixty participants were included in the study.

\section{Data Collection}

All participants were interviewed using a well-structured, self-directed questionnaire that included the ESR median distribution, the relationship between RA serotypes and DAS28-ESR, joint involvement and distributions, the relationship between other immune diseases and RA, the relationship between DAS28-ESR and the duration of the disease, the use of disease-modifying antirheumatic drugs among the participants, comorbidities among the participants, and the presence of extra-articular manifestations among the patients. All participants' disease activity status was assessed by DAS28-ESR. It has four categories: remission if $<2.6$, low activity if $\geq 2.6-<3.2$, moderate activity if $\geq 3.2-\leq 5.1$ and high activity if $>5.1$, with a total score ranging between 0-9.1.

\section{Variables}

Demographic data, age ivided into five main groups as follows: less than 20 years, 20-35 years, 35-50 years, 51-70 years, above 70 years, according to the geographic region into central, eastern, western and northern Sudan, gender (male and female), duration of the disease (less than one year, 1-5 years, more than 5 years), were obtained. Laboratory data were obtained from participants' records: ACPA, IgM RF, ESR.

\section{Data Analysis}

Following data collection, a master sheet was performed. Data were then analyzed using a statistical package of social sciences (SPSS) for windows version 23. Descriptive statistics were performed using frequency tables, histograms and pie charts. The relationship between the positive and negative serotypes and other remaining study variables was tested using the Chi-square test. Yielded results were considered statistically significant at a P-value $<0.05$. 
Table I The Relationship Between the RA Serotypes and the Disease Activity Score-28 for Rheumatoid Arthritis with ESR (DAS28ESR)

\begin{tabular}{|c|c|c|c|c|c|c|}
\hline & & \multicolumn{2}{|c|}{ SPRA (37) } & \multicolumn{2}{|c|}{ SNRA (23) } & \multirow[t]{2}{*}{ P value } \\
\hline & & No & $\%$ & No & $\%$ & \\
\hline \multirow[t]{4}{*}{ DAS28-ESR } & Remission & 3 & $8.1 \%$ & 7 & $30.4 \%$ & \\
\hline & Low disease activity & 4 & $10.8 \%$ & 4 & $17.4 \%$ & 0.004 \\
\hline & Moderate disease activity & 21 & $56.8 \%$ & 12 & $52.2 \%$ & \\
\hline & High disease activity & 9 & $24.3 \%$ & 0 & $0.0 \%$ & \\
\hline
\end{tabular}

\section{Results}

\section{General Characteristics of the Study Population}

This study included 60 participants as per the inclusion criteria; $91.7 \%$ of them were females $(n=55,33$ for SPRA and 22 SNRA) and $8.3 \%$ were males $(n=5,4$ for SPRA and 1 SNRA). Regarding their ages, $38.3 \%$ of the participants were aged between $36-50$ years, followed by $36.7 \%$ who were aged between 51-70 years old and 15\% who were between 20-35 years. The mean age was 48.37 years \pm 13.98 SD. Regarding their origin, most of the participants (41.7\%) were from the western region of Sudan while $26 \%$ and $20 \%$ were from central and northern regions, respectively.

\section{Serotype and Disease Activity}

The distribution of serotype among the patients showed that $61.7 \%(n=37)$ of the participants were seropositive "SPRA" and 38.3\% $(n=23)$ were seronegative "SNRA". The study showed that the majority of the participants (55\%) had a moderate disease activity, followed by $16.7 \%$ who had a remission then $15 \%$ had high disease activity and only $13.3 \%$ had low disease activity.

The DAS28-ESR mean of the participants was $3.9 \pm$ 1.3 SD. Furthermore, it was $4.2 \pm 1.4 \mathrm{SD}$ and $3.3 \pm 1.03$ $\mathrm{SD}$ in seropositive and seronegative groups, respectively. The relationship between the serotypes and DAS28-ESR was examined using the Chi-square test for categorical variables, which yielded results that were considered statistically significant at $\mathrm{P}<0.05$. It was noticed that the seropositive group mostly have either moderate or high disease activity, in contrast to a seronegative group which has more remission and low disease activity. The overall relationship between DAS28-ESR and the serotypes findings was found to be statistically significant at $\mathrm{P}=0.004$ (Table 1). Regarding joint involvement, 43 participants had - at least - one joint tenderness with a mean of 5 joints \pm 6.9 SD. And 16 had at least one swollen joint with a mean of 1 joint \pm 1.9 SD. In more detail, $81.1 \%$ of the seropositive group had joint tenderness and $56.5 \%$ in the seronegative group, while $37.8 \%$ of the seropositive group had swollen joints and only $8.7 \%$ in the other group.

The most frequently affected joints in this study were shoulders ( $\mathrm{n}=27,20$ for SPRA and 7 SNRA), knees $(\mathrm{n}=$ 24, 17 for SPRA and 7 SNRA) and wrists ( $\mathrm{n}=23,16$ for SPRA and 7 SNRA). These were followed by MCP $(\mathrm{n}=$ 18, 15 for SPRA and 3 SNRA) then PIP and elbow ( $\mathrm{n}=$ 10, 7 for SPRA and 3 SNRA) for each. Regarding the relationship between the serotypes and particular joints involvement, only MCP was found to be statistically significant $(\mathrm{P}=0.019)$. It is associated more with the seropositive group. The remaining joints did not show any statistical significance $(\mathrm{P}>0.05)$ (Table 2$)$.

The overall ESR median was $35 \pm 28.9$ SD. When comparing the ESR levels between the two groups, in SPRA the median was $40 \pm 28.7 \mathrm{SD}$ and $30 \pm 26.8 \mathrm{SD}$ in SNRA. Although the ESR median was found to be higher in the SPRA group $(40 \pm 28.9)$ than in SNRA (30 \pm 26.7 ), the association is not statistically significant (Figure 1).

Regarding the duration of the disease, $33.3 \%$ had RA for less than 1 year, while $40 \%$ had it for $1-5$ years and $26.7 \%$ for more than 5 years. There was no significant association between the duration of the disease and RA serotypes $(\mathrm{P}=0.076)$ (Table 3$)$.

DAS28-ESR was found to be statistically significantly related to the duration of the disease $(\mathrm{P}=0.005)$, as the early stages of the disease are more active (Table 4 ).

In regards to medication use, 52 participants were using oral prednisolone, while 49 were taking HCQ and only 26 participants were prescribed methotrexate. The statistical association between DMARDs and RA serotypes is illustrated in Table 5 . 
Table 2 Shows the Distribution of the Joints Manifestations According to the RA Serotypes

\begin{tabular}{|c|c|c|c|c|c|c|}
\hline & & \multicolumn{2}{|c|}{ SPRA (37) } & \multicolumn{2}{|c|}{ SNRA (23) } & \multirow[t]{2}{*}{$P$ value } \\
\hline & & No & $\%$ & No & $\%$ & \\
\hline \multirow[t]{6}{*}{ Joints } & Shoulder & 20 & $54.1 \%$ & 7 & $30.4 \%$ & 0.071 \\
\hline & Elbow & 7 & $18.9 \%$ & 3 & $13 \%$ & 0.547 \\
\hline & Wrist & 16 & $43.2 \%$ & 7 & $30.4 \%$ & 0.318 \\
\hline & Knee & 17 & $45.9 \%$ & 7 & $30.4 \%$ & 0.229 \\
\hline & $\mathrm{MCP}$ & 15 & $40.5 \%$ & 3 & $13 \%$ & 0.019 \\
\hline & PIP & 7 & $18.9 \%$ & 3 & $13 \%$ & 0.547 \\
\hline
\end{tabular}

Considering co-morbidities, $33.3 \%$ of the participants had HTN and $13.3 \%$ were diabetics. Allergic conditions were found in $20 \%$ of them and other autoimmune diseases in $4.7 \%$. Also, there was no statistically significant association between co-morbid diseases and RA serotypes (Table 6).

The most frequent extra-articular manifestation was the ocular symptoms (37\% and 34.8\%) for SPRA and SNRA, respectively. Respiratory symptoms were less frequent $(5.4 \%$ of SPRA and $8.7 \%$ of SNRA). Renal impairment was reported in $2.7 \%$ in SPRA and none in SNRA. However, there was no statistical association between these extraarticular manifestations and the RA serotypes (Table 7).

\section{Discussion}

In recent years, the significance of serological status for diagnosis, treatment, clinical phenotyping and follow-up of RA patients has been well recognized. Identification of ACPA and RF has facilitated recognition of a somewhat homogeneous subgroup of patients with certain environmental and genetic risk factors and knowledge of the severe course of the disease. ${ }^{10}$ In this study, patients were mainly females (91.7\%), aged between $36-50$ years (38.3\%). The profile of age and sex distribution is consistent with previous studies, ${ }^{11}$ this shows the similarities among the African RA patients. In regard to serology, $61.7 \%$ of the participants were found to be seropositive. Some reports indicate the same result as they showed $52 \%$ of seropositive patients. ${ }^{12}$

Some studies highlighted a pattern of increased SPRA among patients in western African countries compared with east and central Africa, the same result has been reported in our study. ${ }^{12}$ In contrast, this percentage is higher among Caucasians compared with African patients at almost $80 \% .^{13}$ This may shed light on the contribution of environmental and genetic factors.
In our study we used the DAS28-ESR score for disease activity, the DAS mean was $3.9 \pm 1.3 \mathrm{SD}$ which is less than the $5.5 \pm 1.6$ yielded from other comparable studies.14,15 This may be explained by the current health condition of the patients as most of them are affected by the drug shortage which imposes an effect on the severity of the disease, and poor rheumatologic care in Sudan.

The relationship between disease activity and serological status is still controversial. In this study, a strong association was established between DAS score and serological status and the DAS28 was higher among the SPRA group, although a few studies did not find a significant association. ${ }^{16}$ This may be explained by the duration from the onset of the disease to the time of presentation to the clinic. Another report found that in early arthritis DAS would be higher among the seronegative group. ${ }^{17}$ This could be attributed to diagnosis delay as the seronegative patients require more clinical symptoms to be classified as having RA according to the $2010 \mathrm{ACR} /$ EULAR criteria.

The relationship between DAS and disease-related symptoms are variable. Out of the 28 joints assessed by DAS28, MCP was found to be the only joint that is significantly associated with DAS28 and its involvement was greater among seropositive patients, this is contrary to other studies which found a significant association between MCP involvement and SNRA. ${ }^{7,9}$ Involvement of MCP can be assessed clinically and may indicate seropositivity and its associated ramifications such as joint destruction, poor prognosis and disability.

The overall ESR median value was found to be $35 \pm 28.9$ SD, this was inconsistent with the median of other studies ${ }^{18}$ which was $56 \mathrm{~mm} / \mathrm{h}$; this gives an idea about the trend of ESR among the studied patients. Still, the difference could be 


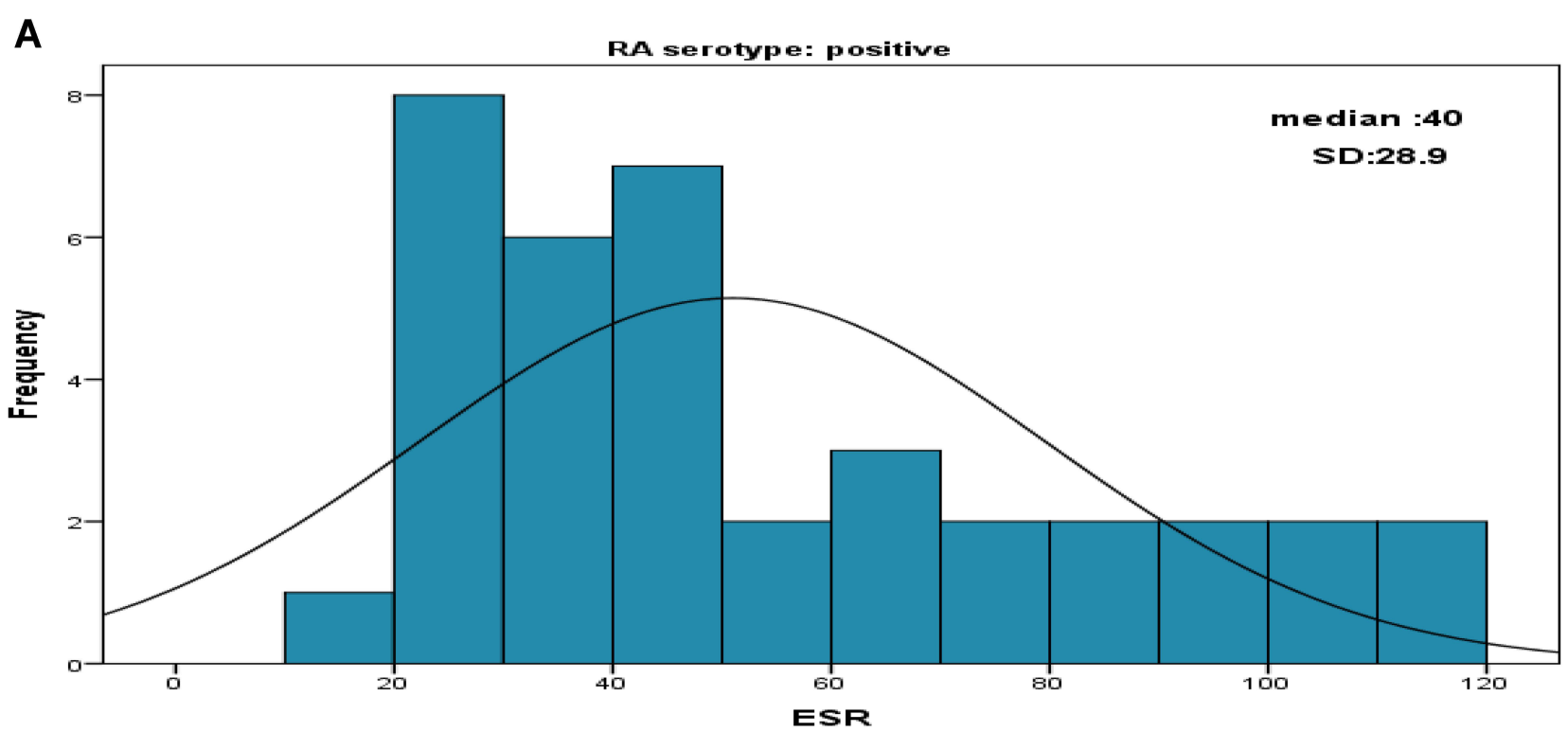

B

RA serotype: negative

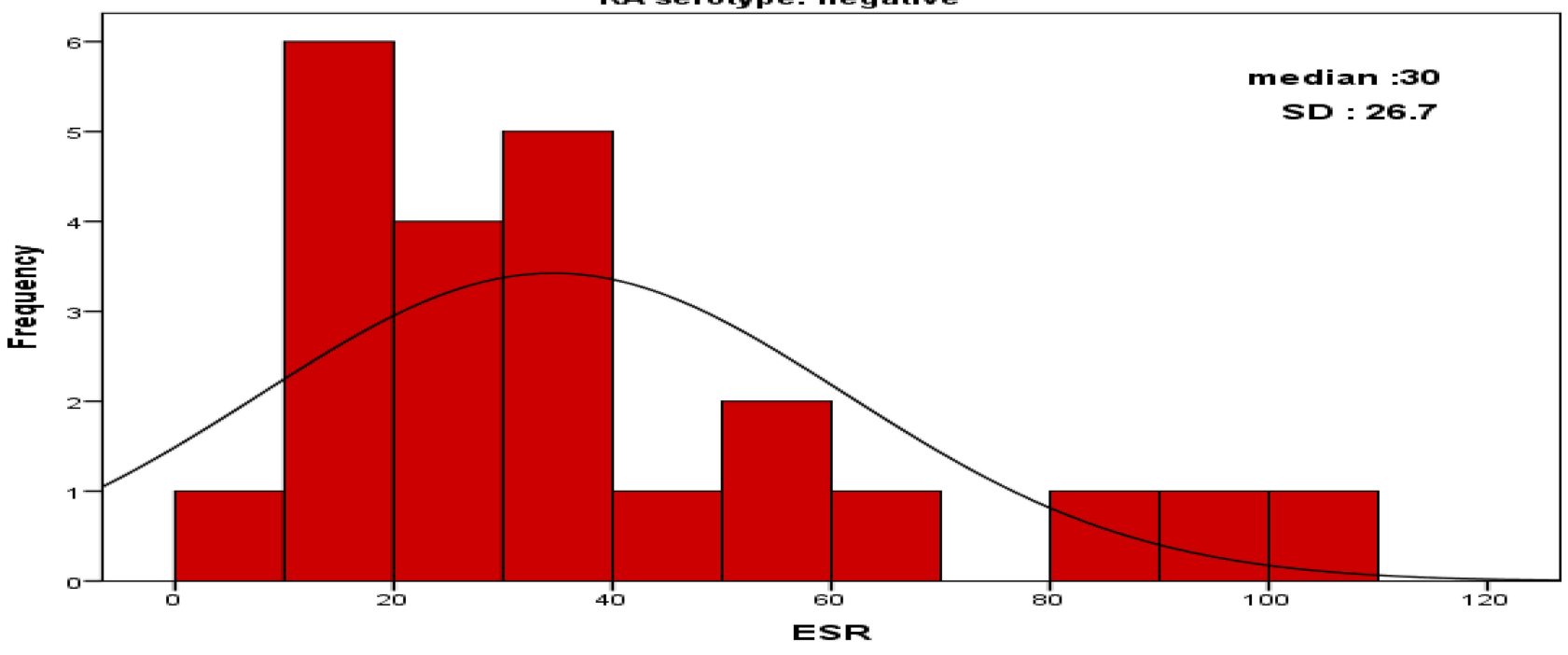

Figure I Shows the ESR distribution among the patients, (A) The ESR median among the SPRA patients, (B) ESR median among the SNRA patients.

attributed to the sample size. In spite of the difference between the overall medians, serotype was not found to be related to ESR median in both studies, which may reflect the contribution of ESR level on DAS28 score.

This study did not find any significant correlation between the presence of extra-articular manifestations and the serological status of RA. However, others found some association between ocular involvement and seropositivity of RA; they reported a strong point that this manifestation was assessed by an experienced ophthalmologist. ${ }^{19}$ This study carries some limitations, firstly the sample size, this was attributed to the COVID19 pandemic associated difficulties related to decreased access to patients during this period. Secondly, the global patient assessment included in DAS is poorly expressed by the patients. 
Table 3 Shows the Distribution of Duration of the Disease Among the Study Participants According to RA Serotypes

\begin{tabular}{|l|l|l|l|l|l|l|}
\hline \multicolumn{2}{|c|}{} & \multicolumn{2}{l}{ SPRA (37) } & \multicolumn{2}{l}{ SNRA (23) } & \multirow{2}{*}{ Total } \\
\cline { 3 - 8 } \multicolumn{2}{|c|}{} & NO & $\%$ & NO & $\%$ & \\
\hline \multirow{2}{*}{$\begin{array}{l}\text { Duration of the } \\
\text { disease }\end{array}$} & $<1$ year & 9 & $24.3 \%$ & 11 & $47.8 \%$ & 20 \\
\cline { 2 - 8 } & $1-5$ years & 15 & $40.5 \%$ & 9 & $39.1 \%$ & 24 \\
\cline { 2 - 8 } & $>5$ years & 13 & $35.1 \%$ & 3 & $13 \%$ & 16 \\
\hline
\end{tabular}

Table 4 Shows the Relationship Between DAS28-ESR and Duration of the Disease

\begin{tabular}{|c|c|c|c|c|c|c|c|c|}
\hline & & \multicolumn{2}{|c|}{$<$ I Year } & \multicolumn{2}{|c|}{ I-5 Years } & \multicolumn{2}{|c|}{$>5$ Years } & \multirow[t]{2}{*}{$P$ value } \\
\hline & & NO & $\%$ & No & $\%$ & No & $\%$ & \\
\hline \multirow{4}{*}{$\begin{array}{l}\text { DAS28- } \\
\text { ESR }\end{array}$} & Remission & 0 & $0.0 \%$ & 5 & $20.8 \%$ & 5 & $31.3 \%$ & \\
\hline & Low disease activity & 4 & $20 \%$ & 4 & $16.7 \%$ & 0 & $0.0 \%$ & \\
\hline & Moderate disease activity & 15 & $75 \%$ & II & $45.8 \%$ & 7 & $43.8 \%$ & 0.005 \\
\hline & High disease activity & I & $5 \%$ & 4 & $16.7 \%$ & 4 & $25 \%$ & \\
\hline
\end{tabular}

Table 5 Shows the Distribution of Disease-Modifying Antirheumatic Drugs (DMARDs) Among the Study Participants According to RA Serotypes

\begin{tabular}{|l|l|l|l|l|l|l|}
\hline \multicolumn{2}{|c|}{} & \multicolumn{2}{l}{ SPRA (37) } & \multicolumn{2}{l}{ SNRA (23) } & \multirow{2}{*}{ Total } \\
\cline { 3 - 8 } \multicolumn{2}{|c|}{} & NO & $\%$ & NO & $\%$ & \\
\hline \multirow{3}{*}{ DMARDs } & Prednisolone & 32 & $86.5 \%$ & 20 & $87 \%$ & 52 \\
\cline { 2 - 8 } & HCQ & 28 & $75.7 \%$ & 21 & $91.3 \%$ & 49 \\
\cline { 2 - 8 } & Methotrexate & 20 & $54.1 \%$ & 6 & $26.1 \%$ & 26 \\
\hline
\end{tabular}

Table 6 Describe the Distribution of Co-Morbid Diseases Among the Study Participants According to RA Serotype

\begin{tabular}{|c|c|c|c|c|c|c|}
\hline & & \multicolumn{2}{|c|}{ SPRA (37) } & \multicolumn{2}{|c|}{ SNRA (23) } & \multirow[t]{2}{*}{ Total } \\
\hline & & NO & $\%$ & No & $\%$ & \\
\hline \multirow{4}{*}{$\begin{array}{l}\text { Co-morbid } \\
\text { diseases }\end{array}$} & DM & 5 & $13.5 \%$ & 3 & $13 \%$ & 8 \\
\hline & HTN & 11 & $29.7 \%$ & 9 & $39.1 \%$ & 20 \\
\hline & Allergic conditions & 6 & $16.2 \%$ & 6 & $26.1 \%$ & 12 \\
\hline & $\begin{array}{l}\text { Other autoimmune } \\
\text { diseases }\end{array}$ & 3 & $8.1 \%$ & I & $4.3 \%$ & 4 \\
\hline
\end{tabular}

In conclusion, this study found that there was a significant association between the seropositivity of RA and disease activity in Sudanese patients, and the MCP joint involvement was found to be significantly associated with SPRA. There was no association between serotype and ESR level. The study also found no relation between serotype and extra-articular involvement. 
Table 7 Shows the Distribution of the Extra-Articular Manifestations According to the RA Serotypes

\begin{tabular}{|l|l|l|l|l|l|}
\hline \multicolumn{2}{|c|}{} & \multicolumn{2}{l|}{ SPRA (37) } & SNRA (23) \\
\cline { 2 - 6 } & NO & $\%$ & NO \\
\hline \multirow{2}{*}{$\begin{array}{l}\text { Extra-articular } \\
\text { manifestations }\end{array}$} & Eye & 14 & $37.3 \%$ & 8 \\
\cline { 2 - 6 } & Lung & 2 & $5.4 \%$ & 2 \\
\cline { 2 - 6 } & Kidney & 1 & $2.7 \%$ & 0 \\
\hline
\end{tabular}

\section{Data Sharing Statement}

All the data used in the study are available from the first and corresponding author on reasonable request.

\section{Ethical Approval}

Ethical approval for the study was obtained from federal Ministry of Health Sudan and Sudan medical specializations board.

\section{Consent for Publication}

Consent for publication was obtained.

\section{Written Consent from the Patients}

Signed consent was obtained from all the patients for the publication of this study and the patients were informed about the purpose of the study which complies with the Declaration of Helsinki.

\section{Funding}

No funding was received.

\section{Disclosure}

All authors declare that they have no conflicts of interest for this work and that no funding was received.

\section{References}

1. Guo Q, Wang Y, Xu D, Nossent J, Pavlos NJ, Xu J. Rheumatoid arthritis: pathological mechanisms and modern pharmacologic therapies. Bone Res. 2018;6(1):15. PubMed PMID: 29736302. Pubmed Central PMCID: 5920070. doi:10.1038/s41413-018-0016-9

2. Matsushita M, Yamaji K, Tamura N. Extra-articular manifestations of rheumatoid arthritis. Juntendo Med J. 2020;66(1):21-26. doi:10.14789/jmj.2020.66.JMJ19-R01

3. Zhang L, Zhang Y, Pan J. Immunopathogenic mechanisms of rheumatoid arthritis and the use of anti-inflammatory drugs. Intractable Rare Dis Res. 2021;10(3):154-164. doi:10.5582/irdr.2021.01022

4. Vecellio M, Hake VX, Davidson C, Carena MC, Wordsworth P, Selmi C. The IL-17/IL-23 axis and its genetic contribution to psoriatic Arthritis. Front Immunol. 2020;11:3418.
5. Syversen SW, Goll GL, van der Heijde D, et al. Prediction of radiographic progression in rheumatoid arthritis and the role of antibodies against mutated citrullinated vimentin: results from a 10-year prospective study. Ann Rheum Dis. 2010;69(2):345-351. PubMed PMID: 19648126. doi:10.1136/ard.2009.113092

6. Bay-Jensen AC, Siebuhr AS, Damgaard D, et al. Objective and noninvasive biochemical markers in rheumatoid arthritis: where are we and where are we going? Expert Rev Proteomics. 2021;18 (3):159-175. doi:10.1080/14789450.2021.1908892

7. Park E-J, Jeong W, Kim J. Prognostic factors for radiographic progression in patients with seronegative rheumatoid arthritis. $J$ Pers Med. 2021;11(3):184. doi:10.3390/jpm11030184

8. de Punder YM, Hendrikx J, den Broeder AA, Pascual EV, van Riel PL, Fransen J. Should we redefine treatment targets in rheumatoid arthritis? Low disease activity is sufficiently strict for patients who are anticitrullinated protein antibody-negative. J Rheumatol. 2013;40 (8):1268-1274. doi:10.3899/jrheum.121438

9. Nordberg LB, Lillegraven S, Lie E, et al. Patients with seronegative RA have more inflammatory activity compared with patients with seropositive RA in an inception cohort of DMARD-naïve patients classified according to the 2010 ACR/EULAR criteria. Ann Rheum Dis. 2017;76(2):341-345. doi:10.1136/annrheumdis-2015-208873

10. Fujita $\mathrm{Y}$, Asano T, Matsuoka N, et al. Differential regulation and correlation between galectin-9 and anti-CCP antibody (ACPA) in rheumatoid arthritis patients. Arthritis Res Ther. 2020;22(1):1-9. doi:10.1186/s13075-020-02158-3

11. Ouédraogo -D-D, Singbo J, Diallo O, Sawadogo SA, Tiéno H, Drabo YJ. Rheumatoid arthritis in Burkina Faso: clinical and serological profiles. Clin Rheumatol. 2011;30(12):1617-1621. doi:10.1007/ s10067-011-1831-1

12. Elshafie AI, Elkhalifa AD, Elbagir S, et al. Active rheumatoid arthritis in central Africa: a comparative study between Sudan and Sweden. J Rheumatol. 2016;43(10):1777-1786. PubMed PMID: 27481904. doi:10.3899/jrheum.160303

13. Vander Cruyssen B, Nogueira L, Van Praet J, et al. Do all anticitrullinated protein/peptide antibody tests measure the same? Evaluation of discrepancy between anti-citrullinated protein/peptide antibody tests in patients with and without rheumatoid arthritis. Ann Rheum Dis. 2008;67(4):542-546. doi:10.1136/ ard.2007.071654

14. Abdel-Nasser A, Mahmoud M, El Mansoury T, Osman A. Anti$\mathrm{CCP} 2$ is an adjunct to, not a surrogate for, rheumatoid factor in the diagnosis of rheumatoid arthritis: diagnostic utility of antiCCP2 antibodies in Egyptian patients with rheumatoid arthritis. Scand J Rheumatol. 2008;37(5):329-336. doi:10.1080/03009740 802116208

15. Zabsonré WJT, Kaboré F, Nanema D, et al. Frequency and factors associated with depression in rheumatoid arthritis in African black patients: case-control study. Open J Rheumatol Autoimmune Dis. 2019;9(2):35-41. doi:10.4236/ojra.2019.92004 
16. Zemri K, Sellam F, Harir N, et al. Is there an association between anti-citrullinated peptide antibodies and the severity of rheumatoid arthritis parameters in Algerian patients? J Drug Deliv Therapeut. 2020;10(4):17-24. doi:10.22270/jddt.v10i4.4201

17. Barra L, Pope JE, Orav JE, et al. Prognosis of seronegative patients in a large prospective cohort of patients with early inflammatory arthritis. $J$ Rheumatol. 2014;41(12):2361-2369. doi:10.3899/jrheum.140082

18. Elshafie AI, Elbagir S, Aledrissy MI, Elagib EM, Nur MA, Rönnelid J. Occurrence of anti-CCP2 and RF isotypes and their relation to age and disease severity among Sudanese patients with rheumatoid arthritis. Clin Rheumatol. 2019;38(6):1545-1553. doi:10.1007/s10067-019-04431-6
19. Yacoub YI, Amine B, Laatiris A, Hajjaj-Hassouni N. Rheumatoid factor and antibodies against citrullinated peptides in Moroccan patients with rheumatoid arthritis: association with disease parameters and quality of life. Clin Rheumatol. 2012;31(2):329-334. doi:10.1007/s10067-011-1820-4

\section{Publish your work in this journal}

Open Access Rheumatology Research and Reviews is an international, peer-reviewed, open access journal publishing original research, reports, editorials, reviews and commentaries on all aspects of clinical and experimental rheumatology in the clinic and laboratory including the following topics: Pathology, pathophysiology of rheumatological diseases; Investigation, treatment and management of rheumatological diseases; Clinical trials and novel pharmacological approaches for the treatment of rheumatological disorders. The manuscript management system is completely online and includes a very quick and fair peer-review system, which is all easy to use. Visit http://www.dovepress.com/testimonials.php to read real quotes from published authors. 